\title{
Clinical high risk for psychosis model in children and adolescents: a joint position statement of ESCAP Clinical Division and Research Academy
}

\author{
Marco Armando $^{1} \cdot$ Paul Klauser $^{2,3} \cdot$ Dimitri Anagnostopoulos $^{4} \cdot$ Johannes Hebebrand $^{5} \cdot$ Carmen Moreno $^{6}$. \\ Alexis Revet $^{7,8} \cdot$ Jean-Philippe Raynaud ${ }^{7,8}$
}

Published online: 7 March 2020

(c) Springer-Verlag GmbH Germany, part of Springer Nature 2020

\section{Introduction}

Psychotic disorders are one of the most severe mental disorders in children, adolescents, and adults. Accordingly, schizophrenia spectrum and other psychotic disorders (SSD) represent the third leading cause of disability-adjusted life years in young people aged 10-24 years worldwide [14]. Their poor outcome is generally associated with an early age of onset and a longer duration of untreated psychosis and illness [24].

To prevent and reduce the burden caused by psychotic disorders, during the past 2 decades, early detection of risk

Marco Armando

marco.armando@unge.ch

1 Department of Psychiatry, Developmental Imaging and Psychopathology Lab, University of Geneva, Geneva, Switzerland

2 Service of Child and Adolescent Psychiatry, Department of Psychiatry, Lausanne University Hospital, Lausanne, Switzerland

3 Center for Psychiatric Neuroscience, Department of Psychiatry, Lausanne University Hospital, Lausanne, Switzerland

4 Medical School, National and Kapodistrian University of Athens, Athens, Greece

5 Department of Child and Adolescent Psychiatry, Psychosomatics and Psychotherapy, LVR Klinikum Essen, University Hospital Essen, University of Duisburg-Essen, Essen, Germany

6 Child and Adolescent Psychiatry Department, Hospital General, Universitario Gregorio Marañón, Madrid, Spain

7 Service Universitaire de Psychiatrie de l'Enfant et de l'Adolescent, CHU de Toulouse, Toulouse, France

8 UMR 1027, Inserm, Université Toulouse III, Toulouse, France for psychosis has been intensively investigated, and in particular, predictive power for early signs of risk has been translated into clinical practice. This intensive research on early detection has led to the development of the clinical high-risk state for psychosis (CHR-P) construct to enable the identification of subjects at enhanced imminent risk of development of a first-episode psychotic disorder [9]. In particular, the attenuated and transient positive symptoms of the ultra-high-risk criteria and the basic symptom criterion "cognitive disturbances" open promising routes to an indicated prevention and have recently been considered by the European Psychiatric Association (EPA) as diagnostic criteria of a psychosis-risk syndrome [33].

This editorial aims to discuss the particularities associated with the implementation of the CHR-P model in child and adolescent psychiatry, and to propose some priority axes and strategies to sustain this integration.

\section{Do the CHR-P criteria take into account the developmental aspects that characterize children and young adolescents?}

Although the incidence rates for psychosis peak around the age of 22 years [20], one-third of psychotic disorders have an onset before age 18 (early onset psychosis; EOP) [23]. In these cases, the need for early detection becomes even more striking, since EOP is considered to have a poorer outcome than adult-onset psychosis (AOP) [3, 27]. Indeed, because EOP mainly occurs during adolescence, a critical developmental period for the attainment of several milestones including education and peer relationships, individuals with EOP often suffer from medium- and long-term social deficits $[8,15]$.

While EOP is considered to be clinically and biologically coherent with AOP, several differences have been pointed 
out. Individuals with EOP show higher rates of premorbid abnormalities, longer duration of untreated psychosis [5], and poorer outcomes $[3,8,15]$. EOP is also characterized by higher levels of auditory hallucinations [5], negative symptoms, bizarre behavior [5, 16, 32], and more severe cognitive deficits [1, 4, 19, 21, 22, 25, 36]. Genetic loci, which modify the age at onset, have been reported [37].

In comparison with adult CHR-P state, children and adolescents (CAD; aged 8-15 years old) with a CHR-P state report a high prevalence of (attenuated) psychotic symptoms (hallucinations) in both general and help-seeking populations, further underscoring age-related peculiarities of CHR-P symptoms [30]. These seem to decrease throughout adolescence [7, 18, 29] and remit spontaneously in about three quarters of CAD with a CHR-P state $[6,11]$. In addition, 1-year transition rates to full-blown psychosis appear to be lower than those observed in the adult population [2]. A word of caution is warranted in light of the less solid evidence base for these findings in CAD.

Indeed, to date, research into the CHR-P states has predominantly been carried out in adults and older adolescents ( $\geq 16$ years old), with little consideration of possible special requirements in CAD [11]. In accordance with this lack of evidence, the recently published EPA guidance on early detection and intervention in CHR-P [33] argues that, due to the insufficient evidence for the psychosis predictive value of CHR criteria in CAD, these criteria should only be used and communicated with utmost care in CAD. Alongside, EPA guidelines state that evidence on the efficacy of psychological and pharmacological interventions in CAD with a CHR-P state is not sufficient to justify primarily preventive interventions. In line with the EPA statement and under consideration of the evidence available to date, it was recently argued that the validity of current risk criteria needs to be examined in and possibly adapted to children, adolescents, and young adults $[2,26,31,35]$.

\section{Do child and adolescent mental health services have specific competencies and resources for early diagnosis and intervention in CHR-P?}

On the basis of what has been written so far, it is not surprising that patients referred to CHR services by child and adolescent mental health services (CAMHs) have a relatively low pre-test risk for psychosis in comparison with all patients undergoing CHR assessment [10, 11]. Despite a lower transition rate to psychosis in young adolescents presenting with a CHR state, there is evidence for the predictive utility of CHR criteria in CAD and efforts should be made to support the development of CHR-P strategies by CAHMS [2].
Moreover, in general, it seems that CHR-P strategies which are currently in place for adults and older adolescents work well for a small fraction of help-seeking individuals, but not for the majority of patients seeking help in secondary mental health care despite the fact that pre-test risk is up to six times higher than in the general population. Indeed, it has been recently highlighted by a clinical register-based cohort study [12] in the UK that mostly first-episode psychosis patients (i.e., 95\%) who previously sought medical advice in a secondary mental health care such as CAMHS were not tagged as CHR-P, but received a diagnosis of a full-blown mental disorder. Interestingly, diagnoses of bipolar mood disorder or acute and transient psychotic disorders were associated with a similar or even higher risk of transition to psychosis than the original CHR-P criteria themselves [12].

More generally, the distribution of the resources in services (CAMHS and AMHS) primarily focuses on children (0-12 years, developmental disorders) and adults ( $>25$ years, tertiary prevention, and chronicity management). This distribution leaves a few resources to devote the activity of prevention and early intervention that targets the adolescent and young adult populations [34].

In this sense, CAMH practitioners have an important role in redefining more global and trans-diagnostic criteria that take into account the specificity of CAMH services and child development.

In addition, the CHR-P topic and the consistent clinical staging approach are not currently a core subject of teaching in most child and adolescent psychiatry training schools.

\section{ESCAP statement on how to improve the early detection of CHR-P in children and adolescents}

We highlight a few selected areas related to CHR-P in children and adolescents that, in our opinion, should be addressed and implemented in the field of CAMHS.

\section{Development and adaptation of specific and developmentally appropriate tools}

As highlighted in the second paragraph, the majority of current CHR-P diagnostic tools have been developed on populations of young adults. In addition, the value and significance of under-threshold psychotic symptoms have been shown to be different in children and young adolescents.

In this sense, to our knowledge, only two tools for early detection of CHR-P have been developed specifically for children and adolescents to be employed as of age 8 . The first one is the Prodromal Questionnaire-brief child version [17], a self-reported questionnaire that has been validated on a population of 3984 CAD (mean age 10.0 years; SE 
0.01). The second one is the schizophrenia proneness instrument, child and youth version (SPI-CY) [13], which is the only validated structured interview for the assessment of CHR-P states in children and adolescents. The instrument was developed and validated in a sample of 64 CAD aged 8-18 years (mean age 16 years) showing good discriminative and validity values.

Overall, this evidence leads us to emphasize the need for more in-depth studies into developmental peculiarities in the early detection and treatment of psychoses with an onset of illness in childhood and early adolescence. To achieve this goal, it seems necessary to distinguish the predictive utility of risk phenomena (e.g., disorganized communication and unusual thought content/delusional ideas) and their severity category (e.g., attenuated psychotic symptoms and brief limited intermittent psychotic symptoms). In this sense, there is a strong need to develop diagnostic tools that consider all aspects of development in CAD, including biological, affective, cognitive, and social characteristics. Clinician scientists, i.e., CAP specialists who engage in both clinical and research activities [28], will be key players in the refinements of early intervention strategies, including CHR-P.

\section{Develop CHR-P model competencies during training and implement the CHR-P model in CAMHS}

Reasons for a lack of clearly defined CHR-P strategies in CAMHS are probably multifactorial, but the fear of generating stigma in CAD and the resistance to the influence of adult psychiatry on CAP are two important parameters. This is reinforced by a dramatic lack of information and training regarding the early intervention strategies. The younger generation of child and adolescent psychiatrists can play an important role in the diffusion of these new developments and paradigm shifts by teaching and mentoring future trainees. Accordingly, the ESCAP Research Academy supported the first residential course on "Assessment and treatment of psychiatric disorders in children and adolescents" which took place in Catania in October 2019 and during which an entire day was devoted to CHR-P. The second edition of this course will take place in Catania in October 2020 (https:// www.escap.eu/index/the-urgent-need-to-interact/2nd-resid ential-course). In addition, the ESCAP Research Academy meeting to take place in Maastricht in 2021 will focus on early intervention strategies (https://www.escap.eu/escap -congresses/).

\section{References}

1. Alaghband-Rad J, McKenna K, Gordon CT et al (1995) Childhood-onset schizophrenia: the severity of premorbid course. J
Am Acad Child Adolesc Psychiatry 34:1273-1283. https://doi. org/10.1097/00004583-199510000-00012

2. Armando M, Pontillo M, De Crescenzo F, Mazzone L, Monducci E et al (2015) Twelve-month psychosis-predictive value of the ultra-high risk criteria in children and adolescents. Schizophr Res 169:186-192

3. Armando M, Pontillo M, Vicari S (2015) Psychosocial interventions for very early and early-onset schizophrenia: a review of treatment efficacy. Curr Opin Psychiatry 28:312-323. https://doi. org/10.1097/YCO.0000000000000165

4. Asarnow RF, Asamen J, Granholm E et al (1994) Cognitive/neuropsychological studies of children with a schizophrenic disorder. Schizophr Bull 20:647-669. https://doi.org/10.1093/schbu $1 / 20.4 .647$

5. Ballageer T, Malla A, Manchanda R et al (2005) Is adolescent-onset first-episode psychosis different from adult onset? J Am Acad Child Adolesc Psychiatry 44:782-789. https://doi. org/10.1097/01.chi.0000164591.55942.ea

6. Bartels-Velthuis AA, van de Willige G, Jenner JA et al (2011) Course of auditory vocal hallucinations in childhood: 5-year follow-up study. Br J Psychiatry J Ment Sci 199:296-302. https:// doi.org/10.1192/bjp.bp.110.086918

7. Brandizzi M, Schultze-Lutter F, Masillo A et al (2014) Selfreported attenuated psychotic-like experiences in help-seeking adolescents and their association with age, functioning and psychopathology. Schizophr Res 160:110-117. https://doi. org/10.1016/j.schres.2014.10.005

8. Breier A, Schreiber JL, Dyer J, Pickar D (1991) National Institute of Mental Health longitudinal study of chronic schizophrenia. Prognosis and predictors of outcome. Arch Gen Psychiatry 48:239-246. https://doi.org/10.1001/archpsyc.1991.0181027005 1007

9. Fusar-Poli P (2017) The clinical high-risk state for psychosis (CHR-P), version II. Schizophr Bull 43:44-47. https://doi. org $/ 10.1093 / \mathrm{schbul} / \mathrm{sbw} 158$

10. Fusar-Poli P, Palombini E, Davies C et al (2018) Why transition risk to psychosis is not declining at the OASIS ultra high risk service: the hidden role of stable pretest risk enrichment. Schizophr Res 192:385-390. https://doi.org/10.1016/j.schres.2017.06.015

11. Fusar-Poli P, Rutigliano G, Stahl D et al (2016) Deconstructing pretest risk enrichment to optimize prediction of psychosis in individuals at clinical high risk. JAMA Psychiatry 73:1260-1267

12. Fusar-Poli P, Rutigliano G, Stahl D et al (2017) Development and validation of a clinically based risk calculator for the transdiagnostic prediction of psychosis. JAMA Psychiatry 74:493-500. https://doi.org/10.1001/jamapsychiatry.2017.0284

13. Fux L, Walger P, Schimmelmann BG, Schultze-Lutter F (2013) The schizophrenia proneness instrument, child and youth version (SPI-CY): practicability and discriminative validity. Schizophr Res 146:69-78. https://doi.org/10.1016/j.schres.2013.02.014

14. Gore FM, Bloem PJN, Patton GC et al (2011) Global burden of disease in young people aged 10-24 years: a systematic analysis. Lancet Lond Engl 377:2093-2102. https://doi.org/10.1016/S0140 $-6736(11) 60512-6$

15. Häfner H (2000) Onset and early course as determinants of the further course of schizophrenia. Acta Psychiatr Scand 102(Suppl):44-48

16. Kao Y-C, Liu Y-P (2010) Effects of age of onset on clinical characteristics in schizophrenia spectrum disorders. BMC Psychiatry 10:63. https://doi.org/10.1186/1471-244X-10-63

17. Karcher NR, Barch DM, Avenevoli S, Savill M, Huber RS, Simon TJ, Leckliter IN, Sher KJ, Loewy RL (2018) Assessment of the prodromal questionnaire-brief child version for measurement of self-reported psychotic like experiences in childhood. JAMA Psychiatry 75(8):853-861. https://doi.org/10.1001/jamapsychi atry.2018.1334 
18. Kelleher I, Connor D, Clarke MC et al (2012) Prevalence of psychotic symptoms in childhood and adolescence: a systematic review and meta-analysis of population-based studies. Psychol Med 42:1857-1863. https://doi.org/10.1017/S00332917110029 60

19. Kenny JT, Friedman L, Findling RL et al (1997) Cognitive impairment in adolescents with schizophrenia. Am J Psychiatry 154:1613-1615. https://doi.org/10.1176/ajp.154.11.1613

20. Kirkbride JB, Fearon P, Morgan C et al (2006) Heterogeneity in incidence rates of schizophrenia and other psychotic syndromes: findings from the 3-center AeSOP study. Arch Gen Psychiatry 63:250-258. https://doi.org/10.1001/archpsyc.63.3.250

21. Kravariti E, Morris RG, Rabe-Hesketh S et al (2003) The Maudsley early onset schizophrenia study: cognitive function in adolescents with recent onset schizophrenia. Schizophr Res 61:137-148. https://doi.org/10.1016/s0920-9964(02)00291-8

22. Kumra S, Wiggs E, Bedwell J et al (2000) Neuropsychological deficits in pediatric patients with childhood-onset schizophrenia and psychotic disorder not otherwise specified. Schizophr Res 42:135-144. https://doi.org/10.1016/s0920-9964(99)00118-8

23. Madaan V, Dvir Y, Wilson DR (2008) Child and adolescent schizophrenia: pharmacological approaches. Expert Opin Pharmacother 9:2053-2068. https://doi.org/10.1517/14656566.9.12.2053

24. Marshall M, Lewis S, Lockwood A et al (2005) Association between duration of untreated psychosis and outcome in cohorts of first-episode patients: a systematic review. Arch Gen Psychiatry 62:975-983. https://doi.org/10.1001/archpsyc.62.9.975

25. Oie M, Rund BR (1999) Neuropsychological deficits in adolescent-onset schizophrenia compared with attention deficit hyperactivity disorder. Am J Psychiatry 156:1216-1222. https://doi. org/10.1176/ajp.156.8.1216

26. Poletti M, Pelizza L, Azzali S, Paterlini F, Garlassi S, Scazza I, Chiri LR, Gebhardt E, Pupo S, Andrea R (2019) Clinical high risk for psychosis in childhood and adolescence: findings from the 2-year follow-up of the ReARMS project. Eur Child Adolesc Psychiatry 28(7):957-971. https://doi.org/10.1007/s0078 7-018-1262-5

27. Rabinowitz J, Levine SZ, Häfner H (2006) A population based elaboration of the role of age of onset on the course of schizophrenia. Schizophr Res 88:96-101. https://doi.org/10.1016/j.schre s.2006.07.007

28. Revet A, Hebebrand J, Bhide S et al (2018) Dual training as clinician-scientist in child and adolescent psychiatry: are we there yet? Eur Child Adolesc Psychiatry 27:263-265. https://doi. org/10.1007/s00787-017-1104-x

29. Schimmelmann BG, Michel C, Martz-Irngartinger A et al (2015) Age matters in the prevalence and clinical significance of ultrahigh-risk for psychosis symptoms and criteria in the general population: findings from the BEAR and BEARS-kid studies. World Psychiatry 14:189-197. https://doi.org/10.1002/wps.20216

30. Schimmelmann BG, Schmidt SJ, Carbon M, Correll CU (2013) Treatment of adolescents with early-onset schizophrenia spectrum disorders: in search of a rational, evidence-informed approach. Curr Opin Psychiatry 26:219-230. https://doi.org/10.1097/ YCO.0b013e32835dcc2a

31. Schimmelmann BG, Schultze-Lutter F (2012) Early detection and intervention of psychosis in children and adolescents: urgent need for studies. Eur Child Adolesc Psychiatry 21:239-241

32. Schothorst PF, Emck C, van Engeland H (2006) Characteristics of early psychosis. Compr Psychiatry 47:438-442. https://doi. org/10.1016/j.comppsych.2006.03.003

33. Schultze-Lutter F, Michel C, Schmidt SJ et al (2015) EPA guidance on the early detection of clinical high risk states of psychoses. Eur Psychiatry J Assoc Eur Psychiatr 30:405-416. https://doi. org/10.1016/j.eurpsy.2015.01.010

34. Signorini G, Singh SP, Boricevic-Marsanic V, Dieleman G, Dodig-Ćurković K, Franic T, Gerritsen SE, Griffin J, Maras A, McNicholas F, O’Hara L, Purper-Ouakil D, Paul M, Santosh P, Schulze U, Street C, Tremmery S, Tuomainen H, Verhulst F, Warwick J, de Girolamo G, MILESTONE Consortium (2017) Architecture and functioning of child and adolescent mental health services: a 28-country survey in Europe. Lancet Psychiatry 4(9):715-724

35. Tor J, Dolz M, Sintes A, Muñoz D, Pardo M, de la Serna E, Puig O, Sugranyes G, Baeza I (2018) Clinical high risk for psychosis in children and adolescents: a systematic review. Eur Child Adolesc Psychiatry 27(6):683-700. https://doi.org/10.1007/s0078 7-017-1046-3

36. Tuulio-Henriksson A, Partonen T, Suvisaari J et al (2004) Age at onset and cognitive functioning in schizophrenia. Br J Psychiatry J Ment Sci 185:215-219. https://doi.org/10.1192/bjp.185.3.215

37. Woolston AL, Hsiao PC, Kuo PH et al (2017) Genetic loci associated with an earlier age at onset in multiplex schizophrenia. Sci Rep 7(1):6486 